\title{
Reflexiones sobre las estimaciones de mortalidad atribuida al consumo de alcohol a nivel poblacional
}

\section{Considerations about population-level alcohol-attributable mortality estimates}

\author{
Sergi Trias-Llimós****. \\ * Centre d'Estudis Demogràfics, Bellaterra. España. \\ ** Escuela de Londres de Higiene y Medicina Tropical, Londres. Reino Unido.
}

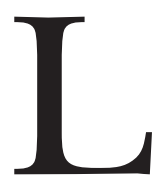
as estimaciones de la mortalidad atribuible al alcohol a nivel poblacional son métricas esenciales para los responsables de las políticas de salud pública. Las causas subyacentes de la muerte proporcionan información importante sobre la mortalidad atribuible al alcohol en las poblaciones, pero en gran medida subestiman el impacto global del alcohol sobre la mortalidad (Rehm et al., 2017; Trias-Llimós, Martikainen, Mäkelä y Janssen, 2018). Pocas causas de muerte se consideran totalmente atribuibles al alcohol (p. ej., hepatopatía alcohólica, trastornos por consumo de alcohol), lo que significa que el alcohol es necesario y que esta muerte no habría ocurrido en ausencia del consumo de alcohol. Sin embargo, para un gran grupo de causas, el alcohol está contribuyendo a la incidencia y el desarrollo de la enfermedad, pero no es un componente necesario (p. ej., cardiopatía isquémica, varios tipos de cáncer) (Rehm et al., 2017). Por tanto, el impacto del alcohol en estas enfermedades no puede obtenerse directamente de los datos de causas subyacentes de muerte y suele estimarse indirectamente.

Los métodos más populares para estimar las estimaciones de la mortalidad atribuible al alcohol a nivel de la población se incluyen dentro de la familia de enfoques de fracción atribuible (FA). El trabajo realizado por Rehm y sus colegas aclaró estas estimaciones desde una perspectiva internacional (Rehm et al., 2007), y fue seguido por los desarrollos de Global Burden of Disease (Stanaway et al., 2018) y por otras publicaciones, incluida la reciente actualización de las estimaciones para España (Donat, Sordo, Belza y Barrio, 2020). En términos generales, estos enfoques requieren dos fuentes diferentes de datos, específicamente: la prevalencia de alcohol específica por edad y sexo y los riesgos relativos ( $R R)$.

La combinación de estas fuentes de datos distintas puede ser problemática, como se discutió anteriormente en otros estudios (Rehm, 2010; Rey y Jougla 2014). Por ejemplo, es bien sabido que una gran parte de la prevalencia de alcohol no se informa en las encuestas de salud y, aunque se aplican correcciones, el alcance del consumo no informado varía de manera impredecible entre los grupos poblacionales, particularmente respecto de los patrones de consumo de alcohol. Además, los riesgos relativos, extraídos de otros análisis, también pueden ser problemáticos. En primer lugar, a menudo se derivan de estudios que evalúan la incidencia de enfermedades y no la mortalidad atribuible a causas específicas. En segundo lugar, los RR son inciertos para determinadas causas y categorías de consumo de alcohol. Por ejemplo, varios estudios todavía usan

Recibido: Septiembre 2020; Aceptado: Diciembre 2020.

Enviar correspondencia a:

Dr. Sergi Trias-Llimós. Centre d'Estudis Demogràfics, Centres de Recerca de Catalunya (CERCA), Carrer de Ca n’Altayó, Edifici E2, Universitat Autònoma de Barcelona, 08193 Bellaterra, España. E-mail: strias@ced.uab.cat. 
RR cardioprotectores mientras que para enfermedades cardiovasculares estos efectos son cuestionados y pueden ser inexistentes (Holmes et al., 2014). En tercer lugar, los RR no suelen estratificarse por grupos de edad, lo que podría desempeñar un papel importante, especialmente para edades avanzadas. Además, se sabe que los RR para condiciones agudas (p. ej., causas externas) tienen una relación inconsistente con los resultados relacionados con el alcohol. A pesar del desarrollo de enfoques específicos para las causas agudas, la estimación del impacto del alcohol en las causas agudas de muerte es compleja e incierta. Estas limitaciones principales tienen un impacto importante en las estimaciones específicas por edad y causa. Por ejemplo, las estimaciones basadas en enfoques de FA suelen sobrestimar potencialmente el impacto del alcohol en la cardiopatía isquémica en la vejez debido al creciente número de muertes a esas edades y al uso de los RR de la edad adulta para la población anciana.

Un enfoque alternativo que puede superar en parte algunas de las limitaciones principales de los enfoques de FA es el que hace una estimación directa de la mortalidad relacionada con el alcohol utilizando los datos detallados de los certificados de defunción, las múltiples causas de muerte (MCOD). Este enfoque presupone que esas muertes con una causa completamente atribuible al alcohol en el certificado de defunción, ya sea como causa subyacente o como causa contribuyente, están relacionadas con el alcohol. Los estudios finlandeses fueron pioneros en adoptar este enfoque para estimar la mortalidad atribuible al alcohol a nivel de la población, y este enfoque se ha adaptado puntualmente en otros países y regiones (Martikainen, Mäkelä, Peltonen y Myrskylä, 2014; Trias-Llimós et al., 2018).

La comparación de los enfoques de FA y MCOD puede resultar en ideas interesantes para identificar las fortalezas y limitaciones de ambos enfoques. Esta comparación es una necesidad esencial para la mayoría de las poblaciones, pero la escasa investigación al respecto sugirió que los enfoques de FA en gran medida sobreestiman la mortalidad atribuible al alcohol en edades más avanzadas (Trias-Llimós et al., 2018), en particular para causas cardiovasculares (Manthey y Rehm, 2019). Además, las estimaciones relacionadas con el alcohol derivadas de los enfoques MCOD parecen representar una parte sustancial de las muertes a edades relativamente jóvenes en Finlandia, lo que parece indicar que este enfoque puede capturar las causas externas de las muertes relacionadas con el alcohol frecuentemente difíciles de estimar (Martikainen et al., 2014).

Utilizar toda la información del certificado de defunción para estimar la mortalidad atribuible al alcohol (enfoques MCOD) requiere afrontar desafíos relacionados con las prácticas de codificación de la mortalidad, a la vez que ofrece nuevas oportunidades para hacer comparaciones y mejorar más las estimaciones. La principal fortaleza de este enfoque es que no requiere ninguna estimación indirecta y, por tanto, la incertidumbre y los posibles sesgos en las estimaciones relacionadas con el enfoque de FA no aplican en ese caso. Los enfoques de MCOD pueden ofrecer nuevos conocimientos sobre la mortalidad relacionada con el alcohol en los grupos de edad en los que los enfoques de FA son más problemáticos, además de aclarar las interrelaciones de causas específicas. Además, existe un número importante de datos de MCOD en varios países desarrollados, y gradualmente aumenta su disponibilidad en otros países, incluido España.

En resumen, los enfoques de FA tienen importantes limitaciones notorias que aplican en particular a las estimaciones relacionadas con el alcohol. Los métodos alternativos que utilizan toda la información del certificado de defunción ofrecen nuevas oportunidades para superar parcialmente esas limitaciones al estimar las muertes relacionadas con el alcohol. Los estudios adicionales deben evaluar específicamente las fortalezas y limitaciones de los enfoques de MCOD para estimar la mortalidad atribuible al alcohol por causas específicas.

\section{Conflicto de intereses}

El autor declara la inexistencia de conflicto de interés.

\section{Referencias}

Donat, M., Sordo, L., Belza, M. J. y Barrio, G. (2020). Mortalidad atribuible al alcohol. En Ministerio de Sanidad, España (2001-2017). Metodología y Resultados. Recuperado de https://pnsd.sanidad.gob.es/profesionales/publicaciones/catalogo/catalogoPNSD/publicaciones / pdf/2020_Mortalidad_atribuible_al_alcohol_en_Espana_2001-2017.pdf.

Holmes, M. V., Dale, C. E., Zuccolo, L., Silverwood, R. J., Guo, Y., Ye, Z.,... on behalf of The InterAct Consortium. (2014). Association between alcohol and cardiovascular disease: Mendelian randomisation analysis based on individual participant data. BMJ 349, 4164-4164. doi:10.1136/bmj.g4164.

Manthey, J. y Rehm, J. (2019). Mortality from alcoholic cardiomyopathy: Exploring the gap between estimated and civil registry data. Journal of Clinical Medicine, 8, 1137. doi:10.3390/jcm8081137.

Martikainen, P., Mäkelä, P., Peltonen, R. y Myrskylä, M. (2014). Income differences in life expectancy: The changing contribution of harmful consumption of alcohol and smoking. Epidemiology, 25, 182-190. doi:10.1097/ EDE.0000000000000064.

Rehm, J., Sulkowska, U., Manczuk, M., Boffetta, P., Powles, J. Popova, S. y Zatonski, W. (2007). Alcohol accounts for a high proportion of premature mortality in Central and Eastern Europe. International Journal of Epidemiology, 36, 458-467. doi:10.1093/ije/dyl294. 
Rehm, J. (2010). Commentary on Rey et al. (2010): How to improve estimates on alcohol-attributable burden? Addiction, 105, 1030-1031.

Rehm, J., Gmel, G. E., Gmel, G., Hasan, O. S. M., Imtiaz, S., Popova, S.,... Shuper, P. A. (2017). The relationship between different dimensions of alcohol use and the burden of disease - an update: Alcohol and disease. Addiction, 112, 968-1001. doi:10.1111/add.13757.

Rey, G. y Jougla, E. (2014). Are alcohol-attributable mortality estimates reliable? European Journal of Public Health, 24, 3-4. doi:10.1093/eurpub/ckt114.

Stanaway, J., Ashkan Afshin, D., Gakidou, E., Lim, S. S., Abate, D. Hassen Abate, K.,... Murray, C. (2018). Global, regional, and national comparative risk assessment of 84 behavioural, environmental and occupational, and metabolic risks or clusters of risks for 195 countries and territories, 1990-2017: A systematic analysis for the Global Burden of Disease study 2017. Lancet, 392, 19231994. doi:10.1016/S0140-6736(18)32225-6.

Trias-Llimós, S., Martikainen, P., Mäkelä, P. y Janssen, F. (2018). Comparison of different approaches for estimating age-specific alcohol-attributable mortality: The cases of France and Finland. PLoS One, 13, e0194478. doi:10.1371/journal.pone.0194478. 
